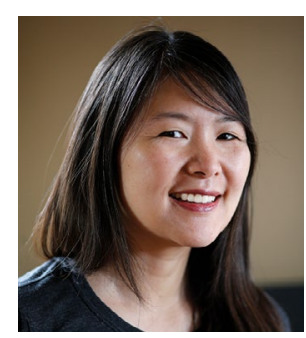

\title{
Using animation to mediate scientific discourse
}

\author{
Interactive annotation and commenting tools provide a means to ground animations in \\ experimental evidence and to support scientific discourse and progress.
}

f you were asked to think about an animation, what is the first thought that pops into your head? A colourful children's story? Or a virus landing on the surface of a cell? It may come as a surprise that the same software tools that bring cartoon characters to life can also serve the scientific community in diverse and important ways.

When I first delved into the field of animation about 15 years ago, animations in molecular and cell biology were largely considered to be a form of eye candy whose main function was to keep students (and faculty!) awake during lectures. However, I had different ideas. During graduate school, and with the support of my graduate advisor, I started to create animations of my lab mates' hypotheses on a part-time basis. Then, as a postdoctoral fellow with my own funding, I made the transition to working on visualization full-time.

My experience in viewing, and later creating, molecular animations convinced me that this form of visualization can play an important role in how scientists understand and communicate complex ideas - a conviction that I still hold today.

Nowadays, I head a group at the University of Utah called the Animation Lab. My lab members work closely with collaborators to create detailed animations that depict specific molecular and cellular hypotheses. These hypotheses are largely informed by experimental data, such as structural data from electron cryomicroscopy or X-ray crystallography, spatiotemporal data from light microscopy and FRET measurements, and information about molecular interactions from biochemical data. Our animations reflect a synthesis and interpretation of diverse data, showing how our collaborators have 'connected the dots' to describe how a molecular process may occur. In some cases, the animations have made my collaborators realize that they do not see eye to eye. During one memorable group meeting, I showed a newly completed animation of a molecular process that was being studied by the lab. While the animation was still playing, one member of the lab suddenly spoke out, saying that he had a completely different idea of how the process occurred. Another lab member, disagreeing with both the animation and their lab mate's proposal, described a third hypothesis. Strikingly, these researchers did not realize that their mental models diverged from those of their peers until they watched the animation. This experience, and numerous others like it, have made me realize the important role that visual representations can play in mediating scientific discourse.

Animations can describe processes in far more detail and with more precision than either spoken or written language. Consider, for example, a statement "Protein A and protein B bind" - and an animation that depicts proteins $\mathrm{A}$ and $\mathrm{B}$ undergoing different breathing states and eventually contacting one another at particular regions, resulting in the proteins quickly locking together and adopting a final conformational state. While everyone may agree on the basic statement, the additional spatiotemporal information included in the animation provides much more room for interpretation, disagreement and discourse. The animation allows scientists to more easily understand and discuss differences in their hypotheses. This, in turn, can lead to ideas for specific experiments to determine which model is more likely to be correct.

One significant issue with molecular animations, however, is a general lack of transparency. When watching an animation, segments that are well informed by experimental data can transition seamlessly to segments that may depict structures or movements that are largely conjectural. While using some visual clues (such as representations to differentiate high- or low-confidence structures) can be helpful, a better solution would be to somehow annotate structures and movements within an animation to describe how it has been informed by existing data. While the annotation of an image is straightforward, annotating an animation - especially one that describes crowded and dynamic three-dimensional structures - poses a substantial technical challenge.

In 2020, these issues were on my mind as the SARS-CoV-2 pandemic closed in. As biologists around the world pivoted to contribute to our understanding of this new virus, their data provided an increasingly clear description of the SARS-CoV-2 virus and how it infects our cells. My group, which had become interested in animating our current understanding of SARS-CoV-2, launched a project to create an animation of the SARS-CoV-2 life cycle. With funding from the National Science Foundation, we started a collaboration with Miriah Meyer (Linköping University) to develop new tools to annotate animations and support community discourse. We have since released animations that describe how SARS-CoV-2 enters and replicates within cells via an interactive interface that allows viewers to click on individual proteins and to view relevant annotations, including references and links. The user interface also allows viewers to ask questions and make comments at different points (spatial and temporal) in the animation. In this way, our tools can support discourse and provide transparency to help the viewer understand which segments of the animation are well supported by experimental evidence, and therefore represent a consensus model, and which segments are more controversial. On the basis of feedback from the community, we hope to revise the animation to reflect our current understanding of the SARS-CoV-2 life cycle. We encourage you to participate in the conversation!

As we gain a deeper understanding of diverse molecular and cellular mechanisms, techniques such as three-dimensional animation are becoming increasingly important as tools to synthesize information and communicate our understanding of complex processes to diverse audiences. With new annotation and commenting capabilities, we hope to engage diverse communities, both within and outside of the research sphere, to explore and ask questions about current hypotheses for molecular-scale events.

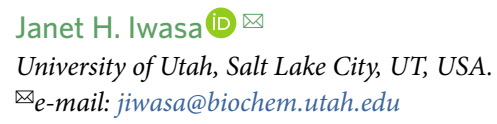

Published online: 20 December 2021 https://doi.org/10.1038/s41564-021-01024-5

Competing interests

The author declares no competing interests. 